\title{
Preditores do Comportamento Antissocial em Adolescentes
}

\author{
Fernanda Lüdke Nardi ${ }^{1}$ \\ Universidade Federal do Rio Grande do Sul \\ Nelson Hauck Filho \\ Universidade São Francisco \\ Débora Dalbosco Dell'Aglio \\ Universidade Federal do Rio Grande do Sul
}

\begin{abstract}
RESUMO - Este estudo investigou preditores do comportamento antissocial em 142 adolescentes em medida socioeducativa de internação (G1) e 691 estudantes de escolas públicas (G2), que responderam a um questionário. Foram observadas diferenças significativas entre os grupos quanto ao comportamento antissocial, violência intra e extrafamiliar, uso de drogas e eventos estressores, com médias mais altas em G1. Dentre as variáveis investigadas, grupo, uso de drogas e eventos estressores, juntamente com a covariável sexo, explicaram $66,5 \%$ da variável dependente. Um modelo de path analysis demonstrou que as variáveis sexo e ambiente estressor, computado pelos eventos estressores, qualidade do relacionamento familiar e violência intra e extrafamiliar predizem o uso de drogas e comportamentos antissociais.
\end{abstract}

Palavras-chave: violência, uso de drogas, comportamento antissocial, eventos estressores

\section{Predictors of antisocial behavior in adolescents}

\begin{abstract}
In this study we investigated predictors of antisocial behavior in 142 young offenders (G1) and 691 public school students (G2), who answered a self-report questionnaire. Significant between-group differences were observed regarding antisocial behavior, intra- and extra-familial violence, drug use and stressor events, with higher scores for G1. The investigated variables, group, drug use, and stressful events, along with the covariate sex, explained $66.5 \%$ of the dependent variable. A path analysis model showed that gender and environmental stressor, computed by stressful events, quality of family relationships and intra and extra familial violence, predict drug use and antisocial behaviors.
\end{abstract}

Keywords: violence, drug use, antisocial behavior, stressor events

Comportamentos antissociais são padrões de conduta intencionais que resultam na agressão física, na agressão relacional ou na violação de normas legal ou moralmente estabelecidas pelo grupo social no qual o indivíduo está inserido (Burt \& Donnellan, 2009). Comportamentos antissociais que se iniciam na infância e na adolescência podem persistir até a fase adulta, facilitando o desenvolvimento de condições psicopatológicas de difícil intervenção (Moffitt, 1993; Pacheco, Alvarenga, Reppold, Piccinini, \& Hutz, 2005). Por exemplo, o Transtorno da Personalidade Antissocial, um padrão recorrente de violação de normas sociais e com reduzidas chances de remissão, é, em geral, precedido por condutas iniciadas antes dos 18 anos (American Psychiatric Association, 2002). Em virtude disso, a literatura psicológica (e.g., Frjick \& Marsee, 2006; Hein, 2004; Neumann, Barker, Koot, \& Maughan, 2010) tem buscado, cada vez mais, investigar a diversidade de fatores de risco individuais, familiares, sociais e culturais que contribuem para a expressão da conduta antissocial em indivíduos jovens, o que é também o foco do presente estudo.

$\mathrm{O}$ estudo das características que distinguem adolescentes em conflito com a lei dos demais adolescentes pode contribuir para elucidar a questão das variáveis que facilitam o surgimento de condutas antissociais. Um estudo mostrou uma predominância, na população de jovens autores de ato

1 Endereço para correspondência: Programa de Pós-Graduação em Psicologia, Universidade Federal do Rio Grande do Sul, Rua Ramiro Barcelos, 2600, sala 115, Porto Alegre, RS, Brasil. CEP: 90.035-003.

E-mail: fernanda.nardi@yahoo.com.br infracional, de indivíduos do sexo masculino, com baixa escolaridade e com histórico de problemas de conduta (Pinho et al., 2006). Em outro estudo, o comportamento antissocial de familiares, os conflitos na família e as práticas educativas parentais se mostraram altamente preditivos do comportamento infrator (Pacheco \& Hutz, 2009). Outros autores também apontam a conduta infracional de familiares como um fator que contribui para o desenvolvimento da delinquência (Loeber \& Dishion, 1983; Nardi, 2010; Patterson, Reid, \& Dishion, 1992). Além disso, aspectos específicos do ambiente familiar, como baixa coesão, presença de conflitos e desafetos na família, têm sido considerados preditores da infração juvenil (Feijó \& Assis, 2004; Nardi, 2010). Esse conjunto de resultados aponta para o sexo masculino, a baixa escolaridade e um contexto de violência e instabilidade familiar como possíveis fatores de risco para a conduta socialmente desviante entre adolescentes.

Além das características do ambiente familiar, a qualidade das relações interpessoais em outros contextos sociais também pode influenciar a manifestação de condutas desviantes em jovens. Eventos estressores e de violência ocorridos em outros ambientes frequentados pelo adolescente (como a escola, por exemplo) podem estimular o desenvolvimento de comportamentos antissociais nos jovens. Em um estudo de Vazsonyi e Flannery (1997), as variáveis escolares, juntamente com as relações familiares, explicaram $40 \%$ da variância dos escores em um instrumento sobre comportamento delinquente. Resultados equivalentes foram relatados por Frias-Armenta, Lopez-Escobar e Diaz- 
Mendez (2003), que verificaram que a conduta antissocial de adolescentes foi influenciada por uma diversidade de variáveis relacionadas tanto a um contexto familiar quanto a um contexto escolar e comunitário negativos. Dessa forma, contextos em que comportamentos agressivos são normativos podem estimular o desenvolvimento de padrões recorrentes de desrespeito e violação das normas sociais. Além disso, um ambiente escolar negativo pode contribuir para o afastamento do jovem do estudo formal, sendo a baixa escolaridade uma das características encontradas em estudos sobre jovens infratores (Loeber \& Dishion, 1983; Oliveira \& Assis, 1999; Silva, 2002).

Outro aspecto potencialmente influente é o uso de drogas. Esse aspecto tem sido apontado por diversos autores como estreitamente relacionado ao comportamento antissocial em adolescentes (Ferrigolo et al., 2004; Mason \& Windle, 2002; McManus, Alessi, Grapentine, \& Brickman, 1984; Nardi, 2010; Pacheco \& Hutz, 2009; Wainer, 2006). De fato, o comportamento de uso de drogas e os comportamentos antissociais, muitas vezes, ocorrem dentro de um contexto de desenvolvimento repleto de eventos estressores. Um estudo longitudinal com 1218 adolescentes mostrou, por exemplo, que o uso de drogas por adolescentes do sexo masculino em conflito com a lei mantinha uma relação de influência mútua com comportamentos delitivos (Mason \& Windle, 2002). Embora a motivação subjacente ao uso de substâncias seja um fenômeno complexo e que varia entre os indivíduos, é possível que o uso de drogas, em diversas situações, seja antecedido por afetos negativos decorrentes de eventos estressores vivenciados (Mason \& Windle, 2002).

Nesse contexto de uso de drogas, relações familiares conflituosas e eventos estressores, as perspectivas futuras dos jovens infratores podem ficar comprometidas. Um estudo brasileiro realizado com uma amostra de adolescentes autores de ato infracional mostrou que as expectativas quanto ao futuro mais almejadas por eles tendem a estar relacionadas mais a ter uma família do que a concluir o ensino escolar (Nardi, Jahn, \& Dell'Aglio, 2014). Em outro estudo, com jovens de diferentes contextos (que viviam com a família, em acolhimento institucional e em medidas socioeducativas), foi observado que os adolescentes que cumpriam medidas de restrição de liberdade apresentavam médias mais baixas nas suas expectativas de futuro, especialmente no que se refere à vida escolar (Zappe, Moura Junior, Dell'Aglio, \& Sarriera). A falta de perspectivas futuras, de acordo com Jacobina e Costa (2007), pode estar relacionada às urgências de suas vidas, que lhes cobram dedicação ao trabalho e ao seu sustento (e, por vezes, ao de suas famílias), comprometendo a realização de seus desejos e os possíveis planos acadêmicos. Em virtude disso, essa variável pode também cumprir um papel como potencial preditor de comportamentos antissociais em adolescentes.

Verifica-se, dessa maneira, que o comportamento antissocial em indivíduos jovens ocorre em função de uma diversidade de variáveis biológicas (como idade e sexo), psicológicas (como uso de drogas e falta de perspectivas futuras) e sociais (como violência intra e extrafamiliar, escolaridade e eventos estressores) (Dodge \& Pettit, 2003). Os modelos teóricos têm proposto que o comportamento antissocial é resultado de uma interação de múltiplas disposições biológicas e condições ambientais específicas (Dodge \& Pettit, 2003; Popma \& Raine, 2006). Em virtude disso, o presente estudo teve como objetivo testar um modelo sobre a contribuição de diversas variáveis psicológicas e contextuais na explicação dos comportamentos antissociais em adolescentes. Além disso, buscou-se investigar diferenças entre adolescentes autores de ato infracional e escolares quanto a uma série de variáveis, bem como a relação parcial entre essas variáveis e o comportamento antissocial, após controlar o efeito da variável do grupo de pertença dos adolescentes.

\section{Método}

\section{Participantes}

Participaram da pesquisa 833 adolescentes divididos em dois grupos. O primeiro constituiu-se de 142 adolescentes em conflito com a lei, que cumpriam medida socioeducativa em regime fechado (internação) nas unidades da Fundação de Atendimento Socioeducativo (FASE), Rio Grande do Sul Brasil. Os participantes tinham entre 12 e 19 anos $(M=17,15$; $D P=1,2$ ), sendo $89,4 \%$ do sexo masculino. A escolaridade desse grupo variou entre a $2^{\mathrm{a}}$ série do Ensino Fundamental e o $3^{\circ}$ ano do Ensino Médio, sendo que a maioria cursava a $6^{\mathrm{a}}$ série $(24,6 \%)$. O segundo grupo foi composto por uma amostra de 691 jovens estudantes de escolas públicas da cidade de Porto Alegre, no sul do Brasil, com idades entre 12 e 19 anos $(M=15,16 ; D P=1,56)$, sendo $61,1 \%$ do sexo feminino. A escolaridade desse grupo variou entre a $6^{a}$ série do Ensino Fundamental e o $3^{\circ}$ ano do Ensino Médio, sendo que a maioria cursava o $1^{\circ}$ ano do Ensino Médio (30,2\%).

\section{Instrumentos}

Os participantes responderam a um questionário Questionário de Juventude Brasileira Versão II (Dell'Aglio, Koller, Cerqueira-Santos, \& Colaço, 2011) - com questões referentes a fatores de risco e de proteção. O questionário continha perguntas em formato de resposta dicotômico e politômico, cujas propriedades psicométricas foram avaliadas em um estudo anterior (Libório \& Koller, 2009). Os aspectos específicos utilizados nas análises do presente estudo são apresentadas a seguir.

Comportamento antissocial. Foi investigado mediante sete itens, respondidos de forma dicotômica $(0=$ Não, 1=Sim): "Envolvimento em brigas com agressão física/ violência contra pessoas", "Destruição de propriedade", "Envolvimento em pichação", "Assaltou alguém", "Roubou algo", "Vendeu drogas" e "Outras" (e.g., porte de arma, homicídio e latrocínio). O escore total foi computado somando-se o número de atividades ilícitas em que o jovem se envolveu.

Violência intra e extrafamiliar. Foram investigadas através dos seguintes itens, respondidos de forma dicotômica ( $0=$ Não, 1=Sim): "ameaça ou humilhação", "soco ou surra", "agressão com objeto", "mexeu no meu corpo contra minha 
vontade" e "relação sexual forçada". O escore total foi computado somando-se o número de respostas positivas.

Expectativas de futuro. A questão sobre as expectativas para o futuro foi baseada no instrumento construído por Günther e Günther (1998), contendo nove itens em escala tipo Likert com cinco opções de resposta, que avaliavam quais as chances que acreditavam ter de terminar o ensino médio, ingressar em uma universidade, ter um emprego, ter uma família, entre outros $(1=$ Chances muito baixas, $5=$ Chances muito altas). O escore total foi computado através da soma dos escores dos itens.

Uso de drogas. As substâncias investigadas foram álcool, cigarro, cola ou solventes, maconha, cocaína, crack e ecstasy. Os itens foram avaliados no formato dicotômico ( 0 =Não, $1=$ Sim $)$ e o escore total foi computado somando-se o número de respostas positivas. No caso dos adolescentes das escolas, a pergunta referia-se ao último ano, enquanto no caso dos jovens da FASE, referia-se ao período antes de ingressar na instituição.

Percepção acerca do relacionamento familiar. Foi investigada através de 15 itens, tais como "meus pais raramente me criticam" e "eu me sinto aceito pelos meus pais", avaliados em escala Likert de cinco pontos $(1=$ Discordo totalmente, $5=$ Concordo totalmente $)$. O escore total foi computado através da soma dos escores dos itens.

Eventos estressores. Foi avaliada a ocorrência de vinte eventos estressores, tais como "alguém em minha casa está desempregado", "já morei na rua", "alguém da minha família está ou esteve preso" e "alguém muito importante pra mim faleceu". Ressalta-se que nenhum dos itens inclui violência. Os itens foram dispostos no formato dicotômico $(0=$ Não, $1=$ Sim) e o escore total foi computado somando-se o número de respostas positivas.

\section{Procedimentos e Considerações Éticas}

O estudo foi aprovado pelo Comitê de Ética em Pesquisa do Instituto de Psicologia da UFRGS (protocolo $n^{\circ} 2009060$ ). Tanto as escolas como a FASE-RS autorizaram a realização da pesquisa. Todos os adolescentes participantes assinaram o Termo de Consentimento Livre e Esclarecido e, para os jovens das escolas, foi solicitado que os pais também o assinassem.

Os dados dos adolescentes da FASE foram coletados em cinco unidades de internação, sendo uma unidade feminina (a única existente). O critério de seleção dos participantes foi escolaridade mínima de $5^{\mathrm{a}}$ série e que pudessem compreender adequadamente os instrumentos. Alguns adolescentes com menor escolaridade, mas que desejaram participar, responderam individualmente ao questionário com o auxílio dos pesquisadores. A aplicação dos instrumentos foi coletiva, em grupos de 6 a 8 jovens, com duração aproximada de 60 minutos.

Para a composição do grupo de adolescentes das escolas, foi constituída uma amostra aleatória por conglomerados através de sorteio das escolas públicas municipais e estaduais, seguido pelo sorteio das turmas. $\mathrm{O}$ número de participantes foi obtido através do cálculo amostral, a partir do número total de alunos de Ensino Fundamental e Ensino Médio matriculados em escolas públicas de Porto Alegre, com uma margem de erro estabelecida de 4\% (Barbetta, 2001). Foram coletados dados em 12 escolas, com uma média de 50 adolescentes em cada uma. A aplicação dos instrumentos foi coletiva, em grupos de 20 a 30 jovens, com duração aproximada também de 60 minutos.

\section{Análise de Dados}

Teste $t$ e testes qui-quadrado foram empregados para avaliar diferenças entre os dois grupos quanto às variáveis do estudo. Correlações de Pearson e uma regressão múltipla foram utilizadas para avaliar o relacionamento linear entre as variáveis. O modelo de path analysis foi testado com o programa Mplus 6.0 (Muthén \& Muthén, 2010), sendo empregado o método de estimação Weighed Least Squares Mean and Variance-Adjusted (Muthén, du Toit, \& Spisic, 1997).

\section{Resultados}

A Tabela 1 apresenta as médias das variáveis por grupo. Pode-se observar que houve diferença significativa $(p<0,05)$ entre os grupos quanto às variáveis violência intra e extrafamiliar, uso de drogas, eventos estressores e comportamento antissocial, sendo que o grupo da FASE apresentou média superior ao grupo das escolas. Já as variáveis percepção de relacionamento familiar e expectativas de futuro, que também mostraram diferenças significativas, apresentaram média superior no grupo das

Tabela 1. Comparações de Médias entre os Grupos para as Variáveis Investigadas

\begin{tabular}{|c|c|c|c|c|c|c|c|}
\hline & \multicolumn{2}{|c|}{ FASE } & \multicolumn{2}{|c|}{ Escolas } & \multirow[b]{2}{*}{$t$} & \multirow{2}{*}{$\begin{array}{c}d \text { de } \\
\text { Cohen }\end{array}$} & \multirow{2}{*}{$p$} \\
\hline & Média & $\mathrm{DP}$ & Média & $\mathrm{DP}$ & & & \\
\hline Violência Intrafamiliar & 1,06 & 1,09 & 0,68 & 0,95 & $-3,71$ & 0,37 & $<0,001$ \\
\hline Violência Extrafamiliar & 1,37 & 1,25 & 0,72 & 0,90 & $-5,46$ & 0,60 & $<0,001$ \\
\hline Relacionamento Familiar & 55,15 & 13,19 & 58,83 & 11,96 & 3,24 & 0,29 & 0,001 \\
\hline Uso de Drogas & 3,25 & 1,73 & 1,09 & 1,03 & $-13,11$ & 1,56 & $<0,001$ \\
\hline Expectativas de Futuro & 3,90 & 0,78 & 4,26 & 0,63 & 5,01 & 0,51 & $<0,001$ \\
\hline Eventos Estressores & 8,72 & 3,70 & 3,33 & 2,10 & $-16,71$ & 1,85 & $<0,001$ \\
\hline Comportamento Antissocial & 3,13 & 1,66 & 0,30 & 0,78 & $-18,85$ & 2,31 & $<0,001$ \\
\hline
\end{tabular}


escolas. Destacam-se as variáveis uso de drogas, eventos estressores e comportamento antissocial, cujo $d$ de Cohen foi superior a 0,8 , indicando que a diferença entre os grupos foi grande. Especificamente, o tamanho do efeito observado para comportamentos antissociais foi $d=2,31$, indicando uma diferença extremamente grande entre os grupos quanto a essa variável.

Com a finalidade de explorar um pouco mais as diferenças entre os grupos quanto ao comportamento antissocial, foram conduzidos testes qui-quadrado para cada um dos sete tipos de comportamentos antissociais. Observou-se frequência significativamente maior dos comportamentos nos jovens do grupo da FASE.

A seguir, foram realizadas correlações entre as variáveis investigadas, sendo os resultados apresentados na Tabela 3. Podem-se observar correlações significativas entre todas as variáveis com o total de comportamentos antissociais. Destaca-se que variáveis positivas como escolaridade, percepção acerca do relacionamento familiar e expectativas de futuro correlacionaram-se negativamente com o comportamento antissocial. Por outro lado, a variável que apresentou maior correlação foi eventos estressores, seguida de uso de drogas. Também puderam ser observadas outras correlações importantes, tais como entre uso de drogas e eventos estressores $(r=0,46)$, violência intrafamiliar e percepção de relacionamento familiar $(r=-0,36)$, violência extrafamiliar e uso de drogas $(r=0,35)$ e violência extrafamiliar e eventos estressores $(r=0,31)$.

Tabela 2. Testes Qui-quadrado para as Variáveis Grupo e Comportamentos antissociais

\begin{tabular}{lccc}
\hline & $\begin{array}{c}\text { FASE } \\
\mathbf{\%}\end{array}$ & $\begin{array}{c}\text { Escolas } \\
\mathbf{\%}\end{array}$ & $\begin{array}{c}\text { Teste qui- } \\
\text { quadrado }\end{array}$ \\
\hline $\begin{array}{l}\text { Brigas com agressão } \\
\text { física }\end{array}$ & 66,7 & 15 & $\chi^{2}(1)=160,169$ \\
$\begin{array}{l}\text { Destruição de } \\
\text { propriedade }\end{array}$ & 17,5 & 3,7 & $\chi^{2}(1)=36,78$ \\
Pichação & & & \\
Assalto & 20,6 & 6,8 & $\chi^{2}(1)=25,20$ \\
Roubo & 71,4 & 1 & $\chi^{2}(1)=498,327$ \\
Venda de drogas & 57,1 & 2,2 & $\chi^{2}(1)=333,672$ \\
Outros & 69,3 & 1,6 & $\chi^{2}(1)=455,985$ \\
\hline
\end{tabular}

Na sequência, buscou-se avaliar a contribuição parcial de cada uma das variáveis investigadas para a explicação dos comportamentos antissociais após controlar o efeito da variável do grupo de pertença dos adolescentes. Uma vez que os estudos mostram uma influência do sexo biológico, da idade e da escolaridade no comportamento antissocial (e.g., Dodge \& Pettit, 2003; Loeber \& Dishion, 1983; Oliveira \& Assis, 1999; Silva, 2002), essas variáveis foram incluídas na equação de regressão, a fim de remover sua variância compartilhada com as demais variáveis independentes. As demais variáveis foram uso de drogas, violência intra e extrafamiliar, expectativas de futuro, relacionamento familiar e eventos estressores. $\mathrm{O}$ método de análise foi $\mathrm{o}$ enter, sendo excluídos casos outliers com base nos resíduos padronizados maiores do que $|2,00|$, a fim de evitar vieses na estimação dos parâmetros do modelo. Os resultados são apresentados na Tabela 4. O modelo final de regressão foi significativo, $F(11,758)=113,40, p<0,001$, explicando $66,5 \%$ da variância da variável dependente ( $R^{2}$ ajustado). Observaram-se quatro preditores com contribuição parcial significativa para explicação da variável dependente: grupo, $\beta=0,47, p<0,001$; sexo, $\beta=-0,17, p<0,001$; uso de drogas, $\beta=0,09, \mathrm{p}<0,001$; e eventos estressores, $\beta=0,29, p<0,001$. Dessa forma, controlando-se a influência da variável de grupo - detectada previamente nas comparações de médias

Tabela 4. Modelo de Regressão Múltipla Explicando Comportamento Antissocial

\begin{tabular}{|c|c|c|c|c|c|}
\hline Preditores & $F$ & $\begin{array}{c}R^{2} \\
\text { ajustado }\end{array}$ & Beta & $T$ & $p$ \\
\hline & 113,40 & 0,66 & & & $<0,001$ \\
\hline Grupo & & & 0,47 & 11,11 & $<0,001$ \\
\hline Eventos Estressores & & & 0,29 & 8,62 & $<0,001$ \\
\hline Sexo & & & $-0,17$ & $-6,33$ & $<0,001$ \\
\hline Uso de drogas & & & 0,09 & 2,76 & 0,006 \\
\hline Idade & & & $-0,02$ & $-0,70$ & 0,570 \\
\hline Escolaridade & & & 0,00 & 0,02 & 0,987 \\
\hline Violência intrafamiliar & & & 0,01 & 0,20 & 0,840 \\
\hline Violência extrafamiliar & & & $-0,02$ & $-0,59$ & 0,557 \\
\hline Expectativas de futuro & & & $-0,02$ & $-0,68$ & 0,494 \\
\hline $\begin{array}{l}\text { Relacionamento } \\
\text { familiar }\end{array}$ & & & $-0,03$ & $-1,12$ & 0,264 \\
\hline
\end{tabular}

Tabela 3. Correlações entre as Variáveis do Estudo

\begin{tabular}{|c|c|c|c|c|c|c|c|}
\hline & 2 & 3 & 4 & 5 & 6 & 7 & 8 \\
\hline 1. ESCO & 0,01 & $-0,09 *$ & 0,05 & $-0,16^{*}$ & $0,09 *$ & $-0,28 * *$ & $-0,27 * *$ \\
\hline 2. VINT & & $0,36^{* *}$ & $-0,36 * *$ & $0,25 * *$ & $-0,12^{*}$ & $0,27 * *$ & $0,17 * *$ \\
\hline 3. VEXT & & - & $-0,24 * *$ & $0,35 * *$ & $-0,13 * *$ & $0,31 * *$ & $0,25 * *$ \\
\hline 4. FAM & & & - & $-0,21 * *$ & $0,24 * *$ & $-0,18 * *$ & $-0,12 *$ \\
\hline 5. DROG & & & & - & $-0,15^{* *}$ & $0,46^{* *}$ & $0,50 * *$ \\
\hline 6. EXP & & & & & - & $-0,22 * *$ & $-0,19 * *$ \\
\hline 7. EVENT & & & & & & - & $0,63 * *$ \\
\hline 8. ANT & & & & & & & - \\
\hline
\end{tabular}

Nota. ESCO = Escolaridade, VINT = Violência intrafamiliar, VEXT = Violência extrafamiliar, FAM = Percepção de relacionamento familiar, DROG = Uso de drogas, EXP $=$ Expectativas de futuro, EVENT = Eventos estressores, ANT = Comportamentos antissocial ${ }^{*} p<0,01 * * p<0,001$ 
-, o sexo masculino, o uso de drogas e os eventos estressores apresentaram uma contribuição incremental para a explicação do comportamento antissocial.

A seguir, foi testado o modelo de path analysis. $\mathrm{Na}$ análise de regressão múltipla, verificou-se que, em função da multicolinearidade entre os preditores eventos estressores, violência intrafamiliar, violência extrafamiliar e relacionamento familiar, apenas a primeira variável apresentou uma contribuição parcial significativa. Por esse motivo, para a path analysis, foi computado um índice (denominado "Ambiente estressor") resumindo essas quatro variáveis a partir de uma análise de componentes principais. As cargas das variáveis no primeiro componente (único acima de 1,0 e explicativo de $45,08 \%$ da variância total) foram: eventos estressores $=0,70$, qualidade do relacionamento familiar $=-0,57$, violência intrafamiliar $=0,72$ e violência extrafamiliar $=0,71$. O índice de ambiente estressor, assim como a variável sexo, foi especificado como influenciando o uso de drogas e os comportamentos antissociais.

Outras relações teóricas testadas foram de que as variáveis idade e escolaridade influenciariam direta e indiretamente os comportamentos antissociais, sendo os caminhos indiretos mediados pelas expectativas de futuro dos adolescentes e pelo uso de drogas. As expectativas de futuro, por sua vez, influenciariam direta e indiretamente os comportamentos antissociais, sendo o caminho indireto mediado pelo uso de drogas. O modelo apresentou um excelente ajuste aos dados, $\chi^{2}=2,11, g l=2, p=0,35, \mathrm{CFI}=1,000$, TLI $=0,997$, RMSEA $=0,008, \mathrm{IC}=0,000-0,070, p=0,810$. Dentre todos os caminhos especificados, não foram significativas apenas as relações entre as variáveis sexo e idade e o uso de drogas, e entre a variável expectativas de futuro e os comportamentos antissociais.

\section{Discussão}

Os resultados deste estudo indicaram que o grupo de adolescentes que cumpria medida socioeducativa na FASE apresentou médias significativamente superiores quanto a todas as variáveis consideradas de risco - comportamento antissocial, violência intra e extrafamiliar, uso de drogas e exposição a eventos estressores. Já as variáveis consideradas de proteção - relacionamento familiar e expectativas de futuro - apresentaram médias maiores no grupo de jovens das escolas. Esses dados indicam diferenças nos contextos de inserção desses adolescentes. Percebe-se que o primeiro grupo esteve mais exposto a fatores com potencial para causar consequências negativas ao desenvolvimento. Ao mesmo tempo, esse mesmo grupo apresentou uma menor exposição àqueles fatores com potencial de proteção. Analogamente, diversos estudos têm retratado o cenário de risco que envolve a população de jovens em conflito com a lei (Feijó \& Assis, 2004; Hein, 2004; Loeber \& Dishion, 1983; Pacheco \& Hutz, 2009).

As análises correlacionais mostraram que o comportamento antissocial esteve positivamente relacionado à violência intra e extrafamiliar e negativamente relacionado à percepção de relacionamento familiar. Esses resultados foram consistentes com outros encontrados para o contexto

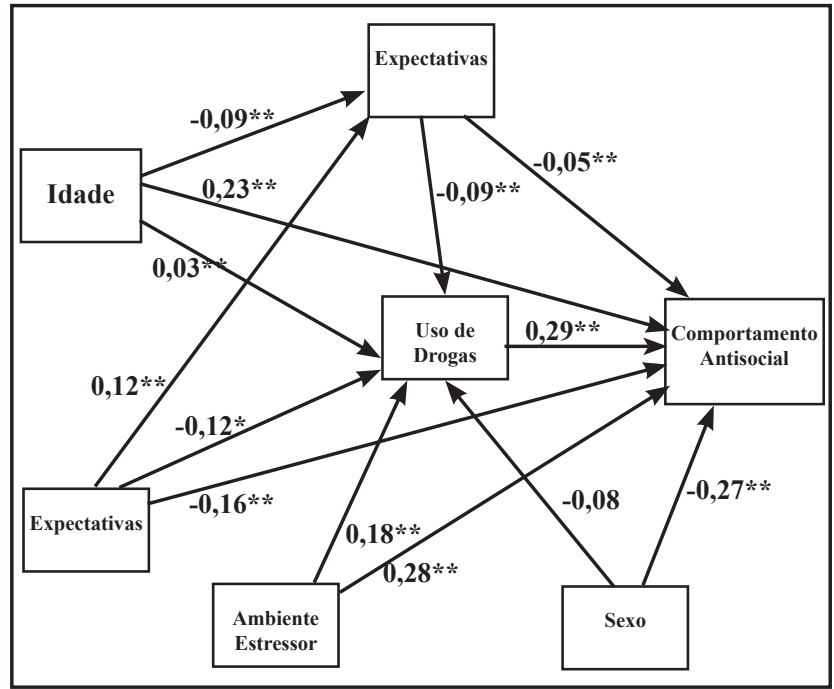

Figura 1. Modelo de Path Analysis

${ }^{*} p>0,05 e^{* *} p>0.01$

brasileiro e internacional (Apel \& Burrow, 2011; Assis, Avanci, Pesce, \& Ximenes, 2009; Fowler, Tompsett, Braciszewski, Jacques-Tiura, \& Baltes, 2009; Hoeve et al., 2009; Lima, Alcântara, Almeid, \& Alves, 2006; Overstreet, 2000; Sá, Curto, Bordin, \& Paula, 2009; Paton, Crouch, \& Camic, 2009). Entretanto, as três variáveis, relacionamento familiar, violência intrafamiliar e violência extrafamiliar, não apresentaram uma contribuição parcial significativa para a explicação do comportamento antissocial (Tabela 4) ao controlar-se a variância compartilhada com a variável grupo. De maneira similar, a escolaridade se mostrou negativamente correlacionada ao comportamento antissocial $(\mathrm{r}=-0,27)$, mas não apresentou explicação parcial na equação de regressão. Essa falta de significância estatística sinaliza uma sobreposição entre essas variáveis e a variável grupo (escolares versus em conflito com a lei). Ou seja, as diferenças entre os grupos podem ser atribuídas às vivências em um contexto de violência, baixa escolaridade, além de instabilidade dentro e fora do ambiente familiar.

Quanto às expectativas de futuro, houve uma correlação negativa com o comportamento antissocial $(\mathrm{r}=-0,19)$, ilustrando a importância da presença de variáveis protetoras como uma forma de amenizar a influência de um contexto negativo, aspecto discutido pela literatura (Muller, Barboza, Oliveira, Santos, \& Paludo, 2009). Lodewijks, Ruiter e Doreleijers (2010) ressaltaram, em sua pesquisa, que poucos estudos têm focado na desistência do comportamento antissocial e nos fatores que auxiliariam essa desistência. Uma das conclusões dos autores foi de que os fatores de proteção auxiliam a diminuição dos efeitos dos fatores de risco que agem sobre os adolescentes. De acordo com Costa e Assis (2006), possuir expectativas de futuro pode contribuir para o fortalecimento do senso de identidade pessoal, conferindo maior nitidez sobre quem se é e o que se deseja, não se deixando influenciar somente pela percepção de outros. Todavia, no presente estudo, após remover a influência das demais variáveis, as expectativas de futuro não apresentaram uma relação negativa significativa que explicasse os comportamentos antissociais (Tabela 4). Do mesmo modo, essa variável também não mostrou significância no modelo 
de path analysis (Figura 1), sugerindo uma relação indireta na explicação do comportamento antissocial, visto que apresentou uma relação negativa direta com o uso de drogas $(\beta=-0,09 ; p<0,01)$, que, por sua vez, esteve relacionado diretamente ao comportamento antissocial $(\beta=0,29 ; \mathrm{p}<0,01)$. Assim, possuir expectativas de futuro pode contribuir para o afastamento das drogas, colaborando para o afastamento de comportamentos antissociais. Verificando-se as correlações da Tabela 2, é possível observar que essa variável apresentou uma correlação de $r=0,24$ com a percepção de relacionamento familiar. Ou seja, as expectativas de futuro se mostraram parcialmente relacionadas também a um ambiente familiar estável, de modo que, ao controlar a influência das demais variáveis familiares e contextuais, não foram significativamente preditoras do comportamento antissocial.

Em relação ao sexo, Bordin e Offord (2000) apontaram que ser do sexo masculino seria um fator de risco para o cometimento de atos infracionais. Confirmando essa tendência, não apenas foi observada uma desigualdade na proporção de meninas e meninos autores de ato infracional na amostra de adolescentes que cumprem medida socioeducativa (apenas 10,6\%), mas também a variável sexo se mostrou preditora do comportamento antissocial. A Figura 1 também revelou esse resultado, mostrando que o sexo masculino explicou essa conduta com $\beta=-0,27(p<0,01)$. Um estudo de revisão sobre essa temática mostrou que aspectos sociais e culturais contribuem para a diferença na manifestação de comportamento antissocial entre os sexos (Boots, Wareham, \& Weir, 2011). Por outro lado, há evidências que sugerem que uma porção substancial das diferenças entre homens e mulheres quanto a comportamentos agressivos pode ser explicada por variáveis neurobiológicas e genéticas (Dodge \& Pettit, 2003).

Por sua vez, o uso de drogas e o ambiente estressor, que explicaram o comportamento antissocial $\operatorname{com} \beta=0,29$ e $\beta=0,28(\mathrm{p}<0,01)$, respectivamente, são fatores considerados altamente perturbadores do desenvolvimento do indivíduo. Ambas as variáveis se relacionam intrinsecamente com afetos negativos, favorecendo a conduta antissocial (Mason \& Windle, 2002; Paton et al., 2009; Patton, 1995; Taylor, Malone, Iacono, \& McGue, 2002; Wainer, 2006). De fato, estudos com modelos animais têm sugerido que as experiências de vida estressantes podem afetar o desenvolvimento do cérebro nos primeiros anos de vida (Goozen \& Fairchild, 2006). Outros estudos também sinalizam o papel das experiências negativas no uso de substâncias (Mason \& Windle, 2002; Patton, 1995). Os resultados obtidos entre as variáveis ambiente estressor e uso de drogas, apresentados na Figura 1, corroboram esses estudos. Assim, eventos estressores, uso de drogas e comportamento antissocial são uma tríade de fatores cuja influência recíproca poderia criar um ciclo de "carreiras delitivas", em que o adolescente se envolveria cada vez mais no mundo do crime e das drogas (Wainer, 2006).

O fenômeno da delinquência juvenil, por si só, constituise em uma grave problemática a ser combatida e, quando aliado ao fenômeno do uso de drogas, as possibilidades de intervenção tornam-se mais complexas. No entanto, se o comportamento antissocial for causado, principalmente, pelo uso de substâncias, um tratamento efetivo poderia auxiliar o combate aos dois fenômenos, assim como intervenções que trabalhem com a questão da droga como um problema social (Wainer, 2006).

Assim, a partir dos resultados, percebe-se um contexto que contribui para que os adolescentes em conflito com a lei tornem-se uma população mais vulnerável a consequências negativas em seu desenvolvimento. De acordo com Feijó e Assis (2004), as vulnerabilidades estão associadas a um contexto de exclusão social em que vivem as famílias dos jovens infratores. Na ideia de exclusão, inserem-se várias formas de discriminação, levando a um conjunto de vulnerabilidades difíceis de superar. No mesmo sentido, Estevam, Coutinho e Araújo (2009) mencionam que, geralmente, antes de conhecerem a criminalidade, esses adolescentes sobreviviam em situações de extrema carência afetiva, educacional e material. Portanto, pode-se pensar nesses jovens não só como infratores, mas, principalmente, como vítimas, seja pela violação de direitos, como direito à educação, seja pela vivência em um ambiente estressor.

\section{Considerações Finais}

O objetivo desse estudo foi investigar variáveis preditoras do comportamento antissocial em adolescentes, além de avaliar diferenças entre escolares e adolescentes em conflito com a lei. Os resultados mostraram que houve diferença significativa entre os grupos quanto ao comportamento antissocial, à violência intra e extrafamiliar, ao uso de drogas e aos eventos estressores, sendo que o grupo de adolescentes da FASE apresentou média superior ao grupo de jovens das escolas. Já as variáveis percepção de relacionamento familiar e expectativas de futuro, que também mostraram diferenças significativas, apresentaram média superior no grupo das escolas. Além disso, observou-se que todas as variáveis independentes tiveram uma correlação significativa com o comportamento antissocial. Dentre as variáveis do estudo, o grupo, o uso de drogas e os eventos estressores, juntamente com a covariável sexo, explicaram juntas $66,5 \%$ da variável dependente. $\mathrm{O}$ modelo de path analysis mostrou que, com exceção das expectativas de futuro, todas as outras variáveis se mostraram capazes de predizer, diretamente, o comportamento antissocial.

Os resultados encontrados no presente estudo podem servir para o aprimoramento de programas de prevenção ao comportamento antissocial já existentes (Gallo, 2008; Wainer, 2006). Um resultado importante nesse sentido é que a escolaridade e as expectativas de futuro apresentaram relações lineares negativas com os comportamentos antissociais. Isso sugere a necessidade de investir no desenvolvimento de aspectos positivos que atuem como moderadores da influência negativa já exercida pelos eventos estressores durante o desenvolvimento. De fato, a ênfase nos aspectos saudáveis do desenvolvimento favorece a emergência do potencial positivo, possibilitando a construção de novas perspectivas aos jovens em risco social (Costa \& Assis, 2006).

Uma pesquisa realizada sobre o mapeamento da situação das unidades de execução de medida socioeducativa de privação de liberdade no Brasil revelou que 53\% não possuem quaisquer iniciativas de apoio ao adolescente que 
sai da instituição e retorna ao convívio social, dificultando, assim, a construção dessas novas perspectivas (Silva \& Gueresi, 2003). A partir dessa realidade, o governo do Estado do Rio Grande do Sul, no Brasil, instituiu o Programa RS Socioeducativo, atual Programa de Oportunidades e Direitos (POD), através do Dec. Projeto de Lei no 295/2008 de 18 de Novembro. Esse programa tem como finalidade, entre outras, auxiliar a inserção familiar, educacional e profissional do adolescente e do jovem adulto. Assim, percebe-se a importância cada vez maior de políticas públicas voltadas a essa população, no intuito de fornecer novas perspectivas de vida aos jovens. Da mesma forma, com novas perspectivas, podem-se amenizar os variados fatores responsáveis pelo ambiente desenvolvimental negativo. Uma limitação do presente estudo foi a pequena quantidade de indivíduos do sexo feminino no grupo de jovens em conflito com a lei, o que impossibilitou a avaliação da interação entre o sexo e o grupo dos adolescentes para as comparações de médias com as variáveis investigadas. Futuros estudos poderão aprofundar o estudo das diferenças entre os aspectos psicológicos e sociais de adolescentes com e sem condutas desviantes, tendo em vista diferenças entre os sexos.

\section{Referências}

American Psychiatric Association (APA). (2002). Manual Diagnóstico e Estatístico de Doenças Mentais (4.ed. ver). Porto Alegre: Artmed.

Apel, R., \& Burrow, J. D. (2011). Adolescent victimization and violent self-help. Youth Violence and Juvenile Justice, 9(2), 112-133. doi: 10.1177/1541204010376939

Assis, S. G., Avanci, J. Q., Pesce, R. P., \& Ximenes, L. F. (2009). Situação de crianças e adolescentes brasileiros em relação à saúde mental e à violência. Ciência \& Saúde Coletiva, 14(2), 349-361. doi: 10.1590/S1413-81232009000200002

Boots, D. P., Wareham, J., \& Weir, H. (2011). Gendered perspectives on depression and antisocial behaviors: An extension of the failure model in adolescents. Criminal Justice and Behavior, 38(1), 63-84. doi: 10.1177/0093854810388504

Bordin, I. A. S., \& Offord, D. R. (2000). Transtorno da conduta e comportamento antissocial. Revista Brasileira de Psiquiatria, 22(Supl. II), 12-15. doi: 10.1590/S1516-44462000000600004

Burt, S. A., \& Donnellan, M. B. (2009). Development and validation of the Sub-Types of Antisocial Behavior Questionnaire (STAB). Aggressive Behavior, 35, 376-398. doi: 10.1002/ab.20314

Costa, C. R. B. S. F., \& Assis, S. G. (2006). Fatores protetivos a adolescentes em conflito com a lei no contexto socioeducativo. Psicologia e Sociedade, 18(3), 74-81. doi: 10.1590/S010271822006000300011

Decreto Projeto de Lei ${ }^{\circ}$ 295/2008 de 18 de Novembro. (2008). Diário Oficial da Assembléia Legislativa. Porto Alegre: Poder Executivo do Estado do Rio Grande do Sul.

Dell'Aglio, D. D., Koller, S. H., Cerqueira-Santos, E., \& Colaço, V. F. R. (2011). Revisando o Questionário da Juventude Brasileira: Uma nova proposta. In D. D. Dell'Aglio \& S. H. Koller (Eds.), Adolescência e Juventude: vulnerabilidade e contextos de proteção (pp. 259-270). São Paulo: Casa do Psicólogo.
Dodge, K. A., \& Pettit, G. S. (2003). A biopsychosocial model of the development of chronic conduct problems in adolescence. Developmental Psychology, 39(2), 349-371. doi: 10.1037/00121649.39.2.349

Estevam, I. D., Coutinho, M. P. L., \& Araújo, L. F. (2009). Os desafios da prática socioeducativa de privação de liberdade em adolescentes em conflito com a lei: Ressocialização ou exclusão social? Psico, 40(1), 64-72.

Feijó, M. C., \& Assis, S. G. (2004). O contexto de exclusão social e de vulnerabilidade de jovens infratores e de suas famílias. Estudos de Psicologia, 9(1), 157-166. doi: 10.1590/S1413294X2004000100017

Ferrigolo, M., Barbosa, F. S., Arbo, E., Malysz, A. S., Stein, A. T., \& Barros, H. M. T. (2004). Prevalência do consumo de drogas na FEBEM, Porto Alegre. Revista Brasileira de Psiquiatria, 26(1), 10-16. doi: 10.1590/S1516-44462004000100006

Fowler, P. P., Tompsett, C. J., Braciszewski, J. M., Jacques-Tiura, A. J., \& Baltes, B. (2009). Community violence: A meta-analysis on the effect of exposure and mental health outcomes of children and adolescent. Development and Psychopathology, 21, 227-259. doi: 10.1017/S0954579409000145.

Frias-Armenta, M., Lopez-Escobar, A. E., \& Diaz-Mendez, S. G. (2003). Predictores de la conducta antisocial juvenil: Un modelo ecológico. Estudos de Psicologia (Natal), 8(1), 15-24. doi: 10.1590/S1413-294X2003000100003

Frick, P. J., \& Marsee, M. A. (2006). Psychopathy and developmental pathways to antisocial behavior in youth. In C. J. Patrick (Ed.), Handbook of Psychopathy (pp. 353-374). New York: The Guilford Press.

Gallo, A. E. (2008). Atuação do psicólogo com adolescentes em conflito com a lei: A experiência do Canadá. Psicologia em Estudo, 13(2), 327-334. doi: 10.1590/S141373722008000200015.

Goozen, S. H. M., \& Fairchild, G. (2006). Neuroendocrine and neurotransmitter correlates in children with antisocial behavior. Hormones and Behavior, 50, 647-654. doi: 10.1016/j. yhbeh.2006.06.021.

Günther, I. A., \& Günther, H. (1998). Brasílias pobres, Brasílias ricas: Perspectivas de futuro entre adolescentes. Psicologia: Reflexão e Crítica, 11, 191-207. doi: 10.1590/S010279721998000200003.

Hein, A. (2004). Factores de riesgo y delincuencia juvenil: Revisión de la literatura nacional e internacional. Providencia: Fundación Paz Ciudadana. Retrieved in Sep 04, 2010, from http:/www.pazciudadana.cl/docs/pub_20090623190509.pdf

Hoeve, M., Dubas, J. S., Eichelsheim, V. I., Van der Laan, P. H., Smeenk, W., \& Gerris, J. R. M. (2009). The relationship between parenting and delinquency: A meta-analysis. Journal of Abnormal Child Psychology, 37, 749-775. doi: 10.1007/ s10802-009-9310-8.

Jacobina, O. M. P., \& Costa, L. F. (2007). "Para não ser bandido": Trabalho e adolescentes em conflito com a lei. Cadernos de Psicologia Social e do Trabalho, 10(2), 95-110.

Libório, R., \& Koller, S. H. (2009). Adolescência e juventude: Risco e proteção na realidade brasileira. São Paulo: Casa do Psicólogo.

Lima, I. M. S. O., Alcântara, M. A. R., Almeid, K. V. D., \& Alves, V. S. (2006). Experiências de violência intrafamiliar entre adolescentes em conflito com a lei. Revista Brasileira de Crescimento e Desenvolvimento Humano, 16(2), 16-24. 
Lodewijks, H. P. B., Ruiter, C., \& Doreleijers, T. A. H. (2010). The impact of protective factors in desistance from violent reoffending: a study in three samples of adolescent offenders. Journal of Interpersonal Violence, 25(3), 568-587. doi: $10.1177 / 0886260509334403$.

Loeber, R., \& Dishion, T. (1983). Early predictors of male delinquency: A review. Psychological Bulletin, 94, 68-99. doi: 10.1037/0033-2909.94.1.68.

Mason, W. A., \& Windle, M. (2002). Reciprocal relations between adolescent substance use and delinquency: A longitudinal latent variable analysis. Journal of Abnormal Psychology, 111, 63-76. doi: 10.1037/0021-843X.111.1.63.

McManus, M., Alessi, N. E., Grapentine, W. L., \& Brickman, A. (1984). Psychiatric disturbance in serious delinquents. Journal of the American Academy of Child Psychiatry, 23, 602-615. doi: 1016/S0002-7138(09)60354-X

Moffitt, T. E. (1993). Adolescence-limited and life-course-persistent antisocial behavior: A developmental taxonomy. Psychological Review, 100(4), 674-701. doi: 10.1037/0033-295X.100.4.674.

Muller, F., Barboza, P. S., Oliveira, C. C., Santos, R. R. G., \& Paludo, S. S. (2009). Perspectivas de adolescentes em conflito com a lei sobre o delito, a medida de internação e as expectativas futuras. Revista Brasileira Adolescência e Conflitualidade, 1(1), 70-87.

Muthén, L. K., \& Muthén, B. O. (2010). Mplus: Statistical analysis with latent variables. User's guide. Los Angeles: Muthén \& Muthén.

Muthén, B., du Toit, S. H. C., \& Spisic, D. (1997). Robust inference using weighted least squares and quadratic estimating equations in latent variable modeling with categorical and continuous outcomes (Unpublished technical report). California: University of California.

Nardi, F. L. (2010). Adolescentes em conflito com a lei: percepções sobre familia, ato infracional e medida socioeducativa (Unpublished masther's thesis), Programa de Pós-Graduação em Psicologia, Universidade Federal do Rio Grande do Sul, Porto Alegre, Rio Grande do Sul, Brasil.

Nardi, F. L., Jahn, G. M., \& Dell'Aglio, D. D. (2014). Perfil de adolescentes em privação de liberdade: eventos estressores, uso de drogas e expectativas de futuro. Psicologia em Revista, 20(1), 116-137.

Neumann, A., Barker, E. D., Koot, H. M., \& Maughan, B. (2010). The role of contextual risk, impulsivity, and parental knowledge in the development of adolescent antisocial behavior. Journal of Abnormal Psychology, 199(3), 534-545. doi: 10.1037/ a0019860.

Oliveira, M. B., \& Assis, S. G. (1999). Os adolescentes infratores do Rio de Janeiro e as instituições que os "ressocializam": A perpetuação do descaso. Caderno de Saúde Pública, 15(4), 831-844. doi: 10.1590/S0102-311X1999000400017.

Overstreet, S. (2000). Exposure to community violence: Defining the problem and understanding the consequences. Journal of Child and Family Studies, 9, 7-25. doi: 10.1023/A:1009403530517.

Pacheco, J., Alvarenga, P., Reppold, C., Piccinini, C. A., \& Hutz, C. (2005). Estabilidade do comportamento anti-social na transição da infância para a adolescência: uma perspectiva desenvolvimentista. Psicologia: Reflexão e Crítica, 18(1), 55-61. doi: 10.1590/S0102-79722005000100008.
Pacheco, J., \& Hutz, C. (2009). Variáveis familiares preditoras do comportamento anti-social em adolescentes autores de atos infracionais. Psicologia: Teoria e Pesquisa, 25(2), 213-219. doi: 10.1590/S0102-37722009000200009.

Paton, J., Crouch, W., \& Camic, P. (2009). Young offenders' experiences of traumatic life events: A qualitative investigation. Clinical Child Psychology Psychiatry, 14(1), 43-62. doi: 10.1177/1359104508100135.

Patterson, G., Reid, J., \& Dishion, T. (1992). Antisocial boys. E.U.A.: Castalia Publishing Company.

Patton, L. H. (1995). Adolescent substance abuse: Risk factors and protective factors. Pediatric Clinics of North America, 42(2), 283-293.

Pinho, S. R., Dunningham, W., Aguiar, W. M., Filho, A. S. A., Guimarães, K., Guimarães, K., Almeida, T. R. P., \& Dunningham, V. A. (2006). Morbidade psiquiátrica entre adolescentes em conflito com a lei. Jornal Brasileiro de Psiquiatria, 55(2), 126-130. doi: 10.1590/S004720852006000200006.

Popma, A., \& Raine, A. (2006). Will future forensic assessment be neurobiologic? Child \& Adolescent Psychiatric Clinics of North America, 15, 429-444. doi: 10.1016/j.chc.2005.11.004.

Sá, D. G. F., Curto, B. M., Bordin, I. A. S., \& Paula, C. S. (2009). Exposição à violência como risco para o surgimento ou a continuidade de comportamento antissocial em adolescentes da região metropolitana de São Paulo. Psicologia: Teoria e Prática, 11(1), 179-188.

Silva, D. F. M. (2002). O desenvolvimento das trajetórias do comportamento delinqüente em adolescentes infratores (Unpublished doctoral dissertation), Universidade Federal do Rio Grande do Sul, Porto Alegre, Rio Grande do Sul, Brasil.

Silva, E. R. A., \& Gueresi, S. (2003). Adolescentes em conflito com a lei: Situação do atendimento institucional no Brasil. Brasília: Instituto de Pesquisa Econômica Aplicada. Retrieved in Sep 04, 2011, from http://www.cibera.de/fulltext/4/4698/ pub/td/2003/td_0979.pdf

Taylor, J., Malone, S., Iacono, W. G., \& McGue, M. (2002). Development of substance dependence in two delinquency subgroups and nondelinquents from a male twin sample. Journal of the American Academy of Child and Adolescent Psychiatry, 41, 386-93. doi: 10.1097/00004583-20020400000010

Vazsonyi, A., \& Flannery, D. (1997). Early adolescents delinquent behavior: Associations with family and school domains. Journal of Early Adolescence, 17(3), 271-293. doi:10.1177/0 272431697017003002.

Wainer, F. W. (2006). Prevención social del delito: Pautas para una intervención temprana en niños y jóvenes. Providencia: Fundación Hanns Seidel Stiftung \& Fundación Paze Ciudadana. Retrieved in Aug 28, 2010, from http://www.pazciudadana.cl/ docs/pub_20090618132605.pdf

Zappe, J. G., Moura Junior, J. F., Dell'Aglio, D. D., \& Sarriera, J. C. (2013). Expectativas quanto ao futuro de adolescentes em diferentes contextos. Acta Colombiana de Psicología, 16(1), 91-100.

Recebido em 01.12.2012

Primeira decisão editorial em 04.05.2015

Versão Final em 13.05.2015

Aceito em 05.06.2015 